\title{
Gyrokinetic particle-in-cell simulations of Alfvén eigenmodes in presence of continuum effects
}

\author{
Alexey Mishchenko* \\ Max Planck Institute for Plasma Physics, D-17491 Greifswald, Germany \\ Axel Könies \\ Max Planck Institute for Plasma Physics, D-17491 Greifswald, Germany \\ Roman Hatzky \\ Max Planck Institute for Plasma Physics, D-85748 Garching, Germany
}

(Dated: March 25, 2014)

\begin{abstract}
First-principle gyrokinetic particle-in-cell simulations of a global Toroidal Alfvén Eigenmode (TAE) are undertaken in the presence of a strong coupling with the continuum. Effects of the bulk plasma temperature on the interplay between the TAE and Kinetic Alfvén Waves (KAW) are investigated. A global TAE-KAW structure is identified which appears to be more unstable with respect to the fast ions than a simple (fluid-like) TAE mode.
\end{abstract}

* alexey.mishchenko@ipp.mpg.de 


\section{INTRODUCTION}

Fast particles can destabilize various shear Alfvén waves (SAW) [1]. These unstable perturbations are considered to be the primary candidate for causing fluctuation-induced transport of the fast particles [2]. Destabilization of the SAW occurs if the mode drive resulting from interaction of a shear Alfvén wave with fast particles exceeds damping from various mechanisms, including Landau damping, continuum damping [3-5], radiative damping [6-8], and others. It is known that weakly damped gap modes, such as Toroidal Alfvén Eigenmodes (TAE) [9], can be destabilized relatively easily [10]. There are many types of Alfvén Eigenmodes (AE) in toroidal plasmas, reflecting asymmetries of various magnetic geometries (e. g. there is a very rich spectrum in non-axisymmetric stellarator geometries [11]). Although TAE modes exist in all toroidal devices, in this paper we limit our attention to the TAE modes in tokamak geometry. The tokamak TAE is used here as a representative for Alfvén Eigenmodes in general and the basic results of our investigation could also be of relevance for other types of $\mathrm{AE}$.

In this paper, we focus on how the bulk plasma parameters may affect the properties of TAE modes destabilised by fast ions. For example, it is well known that many AE damping mechanisms are provided by the bulk plasma. For the Landau mechanism, the TAE instability is damped since it resonantly modifies the velocity-space distribution of the bulk ions or electrons. In the case of the continuum or radiative mechanisms, the mode damping appears in the form of a resonant excitation of Alfvén (continuum) waves or Kinetic Alfvén Waves (KAW) at the radial resonant positions [3-5]. In such a case, there is mode conversion and a resulting coupling between the global TAE mode and the Alfvénic continuum. These resonantly excited Alfvén-continuum waves or KAW themselves represent bulk plasma motion and depend, consequently, on the bulk plasma parameters, such as the temperature. There is a tendency of such resonant mode-conversion processes to generate small scales [3-5], both in the real and velocity spaces. Small scales in the real space imply sensitivity of the associated modes (e. g. KAW) to both ion Finite Larmor Radius (FLR) effects (ion kinetics), and the parallel electric field which eventually may make the electron kinetics important. In other words, the interaction between the global Alfvén modes and the SAW continuum occurs in thin resonant layers and the physics associated with these layers is intrinsically kinetic. In this paper, we are interested in a quantitative description 
of the continuum-coupling effect and the associated locally-kinetic physics on the global properties of the TAE mode. We demonstrate numerically that the AE interaction with the continuum is not always limited to the damping processes, continuum or radiative, caused by the mode conversion. Eventually, coupling to the continuum can make the perturbation more unstable.

Historically, the subject of AE interaction with the continuum has been addressed by many researchers. In 1976, an early influential paper [12] by Hasegawa and Chen introduced the idea of the resonant shear Alfén mode conversion into the KAW. An interest in the subject appeared again in the early 1990s when extensive heating capabilities were installed on the big US and European tokamaks which were approaching fusion-relevant (DeuteriumTritium) regimes of operation. By that time, the concepts of the "toroidal gap" and the "TAE mode" had already been considered and basic questions of mode stability with respect to energetic ions were addressed (late 1980s, see Refs. [13, 14]). In 1992-1994, a number of papers, mostly analytical, appeared [3-5] which considered mode conversion and the associated AE (TAE) damping in detail. The perturbative approach was the core of these studies, in which the continuum coupling represented only a small perturbation modifying neither the spatial structure of the mode nor its frequency. In 1995-2000, a number of experiments [15] dedicated to mode conversion were carried out on JET. Recently (2012), similar experimental studies [16] have been repeated there.

The problem of Alfvén dynamics in toroidal plasmas is of great interest in view of ITER approaching operation in the burning plasma regime. During the last decade, a number of global gyrokinetic numerical models have been developed and implemented in order to tackle this problem from 'first principles', taking into account the effect of the bulk plasma nonperturbatively [17-24]). In this paper, we use the global gyrokinetic particle-in-cell (PIC) code GYGLES [20-22] in order to address the continuum coupling effect on the global TAE mode in tokamak plasmas.

The structure of the paper is as follows. In Sec. II, the basic equations and numerical methods are presented. Section III describes our simulations. Conclusions are drawn in Sec. IV. 


\section{BASIC EQUATIONS AND NUMERICAL APPROACH}

The equations to be solved and the numerical approach used here are identical to Ref. [21]. The code solves the gyrokinetic Vlasov-Maxwell system of equations [25]. The distribution function is split into a background part and a perturbation $f_{s}=F_{0 s}+\delta f_{s}$ with $s=i, e, f$ indicating the particle species (bulk plasma ions, electrons and fast particles). The background distribution function is taken to be a Maxwellian. The perturbed distribution function $\delta f_{s}$ is found from the linearized gyrokinetic Vlasov equation:

$$
\frac{\partial \delta f_{s}}{\partial t}+\dot{\mathbf{R}}^{(0)} \cdot \frac{\partial \delta f_{s}}{\partial \mathbf{R}}+\dot{v}_{\|}^{(0)} \frac{\partial \delta f_{s}}{\partial v_{\|}}=-\dot{\mathbf{R}}^{(1)} \cdot \frac{\partial F_{0 s}}{\partial \mathbf{R}}-\dot{v}_{\|}^{(1)} \frac{\partial F_{0 s}}{\partial v_{\|}}
$$

Here, $\left[\dot{\mathbf{R}}^{(0)}, \dot{v}_{\|}^{(0)}\right]$ correspond to the unperturbed gyrocenter trajectories, while $\left[\dot{\mathbf{R}}^{(1)}, \dot{v}_{\|}^{(1)}\right]$ are the perturbations of the trajectories (proportional to the electromagnetic field fluctuations). The equations of motion are $\left(p_{\|}\right.$-formulation [25] employed):

$$
\begin{aligned}
\dot{\mathbf{R}} & =\left(v_{\|}-\frac{q}{m}\left\langle A_{\|}\right\rangle\right) \mathbf{b}^{*}+\frac{1}{q B_{\|}^{*}} \mathbf{b} \times\left[\mu \nabla B+q\left(\nabla\langle\phi\rangle-v_{\|} \nabla\left\langle A_{\|}\right\rangle\right)\right] \\
\dot{v}_{\|} & =-\frac{1}{m}\left[\mu \nabla B+q\left(\nabla\langle\phi\rangle-v_{\|} \nabla\left\langle A_{\|}\right\rangle\right)\right] \cdot \mathbf{b}^{*}
\end{aligned}
$$

with $\phi$ and $A_{\|}$being the perturbed electrostatic and magnetic potentials, $\mu$ the magnetic moment, $m$ the mass of the particle, $B_{\|}^{*}=\mathbf{b} \cdot \nabla \times \mathbf{A}^{*}, \mathbf{b}^{*}=\nabla \times \mathbf{A}^{*} / B_{\|}^{*}, \mathbf{A}^{*}=\mathbf{A}_{\mathbf{0}}+\left(m v_{\|} / q\right) \mathbf{b}$ the so-called modified vector potential, $\mathbf{A}_{\mathbf{0}}$ the magnetic potential corresponding to the equilibrium magnetic field, $\mathbf{B}=\nabla \times \mathbf{A}_{\mathbf{0}}$, and $\mathbf{b}=\mathbf{B} / B$ the unit vector in the direction of the equilibrium magnetic field. The gyro-averaged potentials are defined as usual:

$$
\langle\phi\rangle=\oint \frac{\mathrm{d} \theta}{2 \pi} \phi(\mathbf{R}+\boldsymbol{\rho}),\left\langle A_{\|}\right\rangle=\oint \frac{\mathrm{d} \theta}{2 \pi} A_{\|}(\mathbf{R}+\boldsymbol{\rho})
$$

where $\boldsymbol{\rho}$ is the gyroradius of the particle and $\theta$ is the gyro-phase. The perturbed electrostatic and magnetic potentials are found self-consistently from the gyrokinetic quasineutrality equation and parallel Ampère's law:

$$
-\nabla \cdot\left[\left(\sum_{s=i, f} \frac{q_{s}^{2} n_{s}}{T_{s}} \rho_{s}^{2}\right) \nabla_{\perp} \phi\right]=\sum_{s=i, e, f} q_{s} \delta n_{s},\left(\sum_{s=i, e, f} \frac{\hat{\beta}_{s}}{\rho_{s}^{2}}-\nabla_{\perp}^{2}\right) A_{\|}=\mu_{0} \sum_{s=i, e, f} \delta j_{\| s},
$$

where $\delta n_{s}=\int \mathrm{d}^{6} Z \delta f_{s} \delta(\mathbf{R}+\boldsymbol{\rho}-\mathbf{x})$ is the perturbed gyrocenter density (distinct from the physical density), $\delta j_{\| s}=q_{s} \int \mathrm{d}^{6} Z \delta f_{s} v_{\|} \delta(\mathbf{R}+\boldsymbol{\rho}-\mathbf{x})$ is the perturbed gyrocenter current (distinct from the physical current), $q_{s}$ is the charge of the particle, $\mathrm{d}^{6} Z=B_{\|}^{*} \mathrm{~d} \mathbf{R} \mathrm{d} v_{\|} \mathrm{d} \mu \mathrm{d} \theta$ 
is the phase-space volume, $\rho_{s}=\sqrt{m_{s} T_{s}} /(e B)$ is the thermal gyroradius and $\hat{\beta}_{s}=\mu_{0} n_{s} T_{s} / B^{2}$ is half the "partial" plasma beta corresponding to a particular species. The polarization density is treated in the long-wavelength approximation and finite Larmor radius (FLR) effects are neglected for electrons. The zeroth-order densities of the particle species satisfy the quasineutrality equation $\sum_{s} q_{s} n_{s}=0$ with $s=i, e, f$.

The perturbed part of the distribution function is discretized with markers:

$$
\delta f_{s}\left(\mathbf{R}, v_{\|}, \mu, t\right)=\sum_{\nu=1}^{N_{p}} w_{s \nu}(t) \delta\left(\mathbf{R}-\mathbf{R}_{\nu}\right) \delta\left(v_{\|}-v_{\| \nu}\right) \delta\left(\mu-\mu_{\nu}\right)
$$

where $N_{p}$ is the number of markers, $\left(\mathbf{R}_{\nu}, v_{\| \nu}, \mu_{\nu}\right)$ are the marker phase space coordinates and $w_{s \nu}$ is the weight of a marker. The electrostatic and magnetic potentials are discretized with the finite-element method (Ritz-Galerkin scheme):

$$
\phi(\mathbf{x})=\sum_{l=1}^{N_{s}} \phi_{l} \Lambda_{l}(\mathbf{x}), \quad A_{\|}(\mathbf{x})=\sum_{l=1}^{N_{s}} a_{l} \Lambda_{l}(\mathbf{x})
$$

where $\Lambda_{l}(\mathbf{x})$ are the finite elements (tensor product of B splines $[26,27]$ ), $N_{s}$ is the total number of the finite elements, $\phi_{l}$ and $a_{l}$ are the spline coefficients. A detailed description of the discretization procedure can be found in Refs. [28-32]. We apply the so-called phase factor transform [28] to all perturbed quantities in the code. The integrals over the gyroangle, Eq. (4), are approximated by an N-point discrete sum [31, 33, 34]. The cancellation problem $[29,35]$ is solved using the iterative scheme No. 2 described in Ref. [32]. For the numerical parameters, we choose 1600 radial grid points, 32 poloidal grid points, $N_{i}=10^{7}$ ion markers, $N_{e}=4 \times 10^{7}$ electron markers, and $N_{f}=2 \times 10^{7}$ fast ion markers. A poloidal Fourier filter $8 \leq m \leq 14$ and a single toroidal mode $n=-6$ are employed in the simulations. The number of the gyro-points used for the gyro-average, Eq. (4), varies between 4 and 64 depending on the actual value of the marker's gyroradius. The choice of the numerical parameters is similar to Refs. [20,21] and has been verified with convergence studies.

\section{SIMULATIONS}

In Ref. [36], a successful benchmark has been undertaken among the majority of existing energetic-particle codes in the context of a linear TAE mode, first considered in Ref. [21]. This benchmark has been carried out in the framework of the International Tokamak Physics Activity (ITPA). Perturbative kinetic-MHD hybrid codes (CKA-EUTERPE [37] and others, 
see [36] for references), non-perturbative kinetic-MHD hybrid codes [38, 39] and fully gyrokinetic codes $[21,40]$ (where all the species have been treated kinetically) participated in this benchmark and found good agreement in their results. This raises a question about the degree of numerical complexity required to model the energetic-particle physics in burning plasmas, since the least computationally expensive adequate approach is preferred. The answer to this question depends on the particular physical problem under consideration. Thus, a simple perturbative hybrid approach of CKA-EUTERPE was adequate when addressing the ITPA benchmark [36]. In this paper, however, we will design a more complicated case aiming beyond the limits of the perturbative kinetic-MHD approach while keeping the magnetic geometry and plasma parameters similar to the ITPA benchmark [36]. Understanding such limits is important when deciding which numerical complexity is required when addressing a particular physics.

For that purpose, we consider a minor modification of the magnetic geometry used in Refs. [21, 36]: a large-aspect-ratio tokamak with a circular cross-section which has the minor radius $r_{\mathrm{a}}=1 \mathrm{~m}$, the major radius $R_{0}=10 \mathrm{~m}$, the magnetic field on axis $B_{0}=3 \mathrm{~T}$, and the safety factor profile $q(r)=1.5+\left(r / r_{\mathrm{a}}\right)^{2}$, where $r$ is the minor radius of the plasma. The background plasma profiles (Maxwellian unperturbed distribution function) are chosen to be flat with the ion (hydrogen) density $n_{i}=2 \times 10^{19} \mathrm{~m}^{-3}$, and flat temperatures $T_{i}=T_{e}$. Note that only the safety factor profile has been modified here compared to the ITPA benchmark case [36]. It is plotted in Fig. 1 versus the profile used in Refs. [21, 36]. The corresponding continuum is shown in Fig. 2. It is striking how much more complex the structure becomes when the magnetic shear increases (cf. the continuum plotted in Fig. 1 of Ref. [21] for the ITPA case). As a consequence, one could expect here much stronger coupling between global TAEs and the continuum.

Similar to Ref. [21], we start our simulations initializing the perturbed electron distribution function with the toroidal mode number $n=-6$ and a poloidal spectrum which includes two harmonics with $m=10$ and $m=11$. The velocity dependence of the initial electron distribution function is Maxwellian. The initial perturbed ion distribution function is zero. The radial dependence of the initial perturbed electron distribution function is chosen to be a Gaussian centered at the position $s=0.5$ of the accumulation point corresponding to the toroidicity-coupled poloidal harmonics $m=10$ and $m=11$ (see Fig. 2). Here, $s=\sqrt{\psi / \psi_{\mathrm{a}}}$ with $\psi$ the poloidal flux and $\psi_{\mathrm{a}}$ the poloidal flux on the plasma edge. 
Following further Ref. [21], we choose a Maxwellian for the unperturbed distribution function of the fast particles (deuterium ions), a flat fast-particle temperature $T_{f}$ and the fast particle density given by the expression:

$$
n_{f}(s)=n_{0 f} \exp \left[-\frac{\Delta_{\mathrm{n} f}}{L_{\mathrm{n} f}} \tanh \left(\frac{s-s_{\mathrm{n} f}}{\Delta_{\mathrm{n} f}}\right)\right]
$$

Here, $s_{\mathrm{n} f}=0.5$ is the position of the maximal value of $\kappa_{\mathrm{n} f}=\left|\nabla n_{f}\right| / n_{f}, n_{0 f}$ is the fast particle density at $s=s_{\mathrm{n} f}, \Delta_{\mathrm{n} f}=0.2$ is the "width" of the density profile, and $L_{\mathrm{n} f}=0.3$ is the "gradient length" of the fast-particle density profile.

First, we perform a gyrokinetic PIC simulation using the fast ion density $n_{0 f}=1.5 \times$ $10^{17} \mathrm{~m}^{-3}$ and the fast ion temperature $T_{f}=400 \mathrm{keV}$. For these parameters, the characteristic fast-particle beta is $\beta_{\text {fast }}=0.0027$, computed at $s=0.5$. The characteristic bulk plasma beta is $\beta_{\text {bulk }}=0.016$, computed at $s=0.5$ and for $T_{i}=T_{e}=9 \mathrm{keV}$. In Fig. 3, we study how the bulk-ion temperature affects the frequency of the (K)TAE mode. One sees that the frequency increases with the bulk-ion temperature. A similar dependence would result from the finite-pressure effect (frequency upshift due to acoustic coupling [41]). However, the deviation observed in simulations is larger than this explanation alone would suggest. In Fig. 4, the growth rate dependence on the bulk-ion temperature is shown. One sees that the mode is destabilised by the bulk-ion temperature. This effect is clearly absent in the perturbative kinetic-MHD picture where the bulk plasma temperature does not appear as a relevant quantity. It is also in contradiction with the "continuum/radiative damping intuition", which would suggest a stronger damping at higher bulk plasma temperatures, since these correspond to larger bulk-ion gyro-radii.

As a possible explanation, we hypothesise that the original TAE mode is replaced by a 'global kinetic structure' (with the radial patterns plotted in Fig. 5). This structure builds up when the bulk plasma temperature increases. It consists of the TAE (or Kinetic TAE [6], KTAE) mode and the KAW mode. The dominant poloidal harmonic $m=10$ of the KAW mode is excited by the original $(\mathrm{K}) \mathrm{TAE}$ through the mode conversion at the resonance position $s \approx 0.3$. Here, the $(\mathrm{K}) \mathrm{TAE}$ frequency closely approaches the $m=10$ continuum branch (see Fig. 2). It appears that the KAW part of the global structure starts to absorb the fast ion 'free energy' at the continuum resonance position and grows instead of dissipating the (K)TAE-mode energy into heat as it would in the conventional continuum/radiative damping mechanism. As a result, the global (K)TAE-KAW structure is more efficient in 
tapping the fast ion free energy compared to a single (K)TAE mode since it interacts with a larger fraction of the fast ion density gradient. The mechanism proposed works if the KAW mode is indeed destabilised by the fast particles. This requires the intrinsic KAW damping, caused e.g. by the phase mixing between the neighbouring Alfvén-continuum waves, to be smaller than the fast-particle drive. One could hypothesise that the value of the bulk plasma temperature controls exactly this parameter: increasing the temperature, we decrease the intrinsic KAW damping so that the resulting KAW mode is destabilised. In this case, the continuum/radiative damping can be 'inverted' into growth. The numerical observations presented here call for future analytical consideration.

In Figs. 6 and 7, we plot the dependence of the global-mode frequency and the growth rate on the fast ion temperature $T_{f}$ at the fixed fast ion density $n_{0 f}=1.5 \times 10^{17} \mathrm{~m}^{-3}$. Two cases are considered, with the bulk plasma temperatures $T_{i}=T_{e}=9 \mathrm{keV}$ and $T_{i}=T_{e}=$ $1 \mathrm{keV}$. The gyrokinetic result is then compared with the perturbative hybrid-gyrokinetic computation. In the perturbative hybrid scheme, one uses the ideal-MHD TAE eigenmode and eigenfrequency. The growth rate is then computed from the energy transfer between the fast particles and the wave (see Ref. [37] for details). In Fig. 7, one sees that the non-perturbative growth rate closely agrees with the perturbative hybrid result at low bulk plasma temperature $T_{i}=T_{e}=1 \mathrm{keV}$. This provides a good verification of the gyrokinetic code used here. In contrast, at higher bulk plasma temperature $T_{i}=T_{e}=9 \mathrm{keV}$, the non-perturbative gyrokinetic compound (K)TAE-KAW mode is substantially more unstable than the perturbative hybrid mode based on the single ideal-MHD TAE, since the global (K)TAE-KAW structure is more efficient in tapping the fast ion energy.

This global (K)TAE-KAW structure becomes especially distinct when we perform a scan with respect to the fast ion density, varying $n_{0 f}$, see Eq. (8), at fixed fast ion temperature. At larger fast ion densities, the KAW is so strongly driven that it becomes an Energetic Particle Mode (EPM) with the same dominant $m=10$ poloidal harmonic. The spatial location of the mode maximum is largely determined by the fast ion density profile. As a result, the global (K)TAE-EPM structure appears. In Fig. 8, the mode frequency dependence on $n_{0 f}$ is shown. One sees that the frequency increases with $n_{0 f}$, but not too strongly, remaining in the proximity of the toroidicity-induced gap. In Fig. 9, the dependence of the growth rate is shown. We hypothesise that the KAW mode, whose interaction with the fast ions is weaker, transforms at the higher fast ion densities into the EPM, strongly interacting 
with the fast ions. Both, the low- $n_{0 f}$ KAW and the high- $n_{0 f}$ EPM are non-locally coupled to the global (K)TAE mode. The resulting eigenmode structures are shown in Fig. 10 for different values of the fast ion density $n_{0 f}$. The 'composite' nature of the mode becomes apparent if the fast ion density is increased. One sees clearly the maximum of the harmonic with $m=10$, which corresponds to the KAW/EPM part of the global structure, related to the resonance at $s=0.3$ (cf. the continuum in Fig. 2), and the (K)TAE maximum at the accumulation point $s \approx 0.5$, along with the characteristic TAE-like coupling between $m=10$ and $m=11$ poloidal harmonics at this location. At low fast ion density, the mode is stabilised (Fig. 9) since the fast ion energy is not sufficient for the drive to overcome the intrinsic KAW damping. The eigenmode structure reduces to a single TAE with some mode conversion, which is now a sink of energy, occurring at the resonant location without creating an unstable 'compound' there (see Fig. 10).

Interestingly, a 'pure' EPM mode, with no coupling to the TAE mode, can also be easily created in the system considered. For this purpose, one performs a scan with respect to $T_{f}$ but now at fixed fast ion pressure with $\beta_{\text {fast }}=0.0027$, computed at the position of the maximum fast ion density gradient $s_{\mathrm{n} f}=0.5$. In Fig. 11 showing the frequency dependence on $T_{f}$, one can see how the TAE mode transforms into the EPM mode at lower fast ion temperatures or, equivalently, at higher fast ion densities (since $\beta_{\text {fast }}$ is fixed). A similar trend has been observed in Ref. [21] using the "low-shear" safety factor, plotted in Fig. 1. In Fig. 12, one sees that the growth rate of the EPM mode increases considerably at smaller $T_{f}$ in the case considered here (stronger than in Fig. 7 of Ref. [21]). In the present paper, however, the fast ion density is twice as large as in Ref. [21] and, perhaps more importantly, the spatial location of the continuum resonances is shifted to areas with higher fast ion density gradient, since $\mathrm{d} \omega_{A} / \mathrm{d} r$ is larger for the higher magnetic shear considered in this paper. In Fig. 13, the eigenmode is shown. Its structure is similar to the EPM eigenmode found in Ref. [21]. However, the present mode is wider since it is more strongly driven.

\section{CONCLUSION}

We have considered the Alfvén mode stability in the presence of strong coupling between

the global TAE mode and the shear Alfvén continuum. The role of the bulk plasma temperature has been studied. In contrast to the conventional picture of TAE-KAW coupling, 
leading to the continuum and radiative damping [6-8], it has been seen that the effect of the mode conversion can be destabilising. A global coupled (K)TAE-KAW(EPM) structure can form as a consequence of the mode conversion at the continuum-resonance position where both the original TAE and the excited KAW modes resonantly interact with the fast particles. This global structure appears to be more efficient in tapping the fast ion 'free energy' than the narrower TAE mode. The (K)TAE-EPM instability appears when the KAW mode transforms into an EPM. At low fast ion drive, the KAW mode coupled to the original TAE is stable. In this case, the usual continuum/radiative damping picture is valid. A detailed theoretical assessment of the global kinetic structure observed is of future interest.

It is often assumed that the growth rate can be obtained as a simple superposition of the fast ion drive and the bulk plasma damping. In reality, however, the structure of the TAE eigenmode as well as its frequency can be influenced, sometimes strongly, by coupling to the continuum. As a consequence, the simple perturbative picture does not always apply. For example, the perturbative hybrid kinetic-MHD approach fails in the case considered here.

The simulations described in this paper are based on the magnetic geometry which is very similar to that of the ITPA benchmark [36]. Yet, the physical content appears to be much more complex. Such a combination may be of interest for further verification of numerical codes, focusing on non-ideal and non-perturbative capabilities.

\section{ACKNOWLEDGMENTS}

We acknowledge the support of Per Helander for this work. We thank Robert Wolf and Michael Cole for carefully reading the manuscript. This work was carried out using the HELIOS supercomputer system at the Computational Simulation Centre of the International Fusion Energy Research Centre (IFERC-CSC), Aomori, Japan, under the Broader Approach collaboration between Euratom and Japan, implemented by Fusion for Energy and JAEA. Also, some simulations have been performed on the local cluster in Greifswald, where support of Henry Leyh is appreciated. The project has received funding from the Euratom research 
and training program 2014-2018.

[1] M. N. Rosenbluth and P. H. Rutherford, Phys. Rev. Lett. 34, 1428 (1975).

[2] F. Zonca, S. Briguglio, L. Chen, C. Fogaccia, T. S. Hahm, A. V. Milovanov, and G. Vlad, Plasma Phys. Controlled Fusion 48, B15 (2006).

[3] F. Zonca and L. Chen, Phys. Rev. Lett. 68, 592 (1992).

[4] H. L. Berk, J. W. Van Dam, Z. Guo, and D. M. Lindberg, Phys. Fluids B 4, 1806 (1992).

[5] M. N. Rosenbluth, H. L. Berk, J. W. Van Dam, and D. M. Lindberg, Phys. Fluids B 4, 2189 (1992).

[6] R. R. Mett and S. M. Mahajan, Phys. Fluids B 4, 2885 (1992).

[7] H. L. Berk, R. R. Mett, and D. M. Lindberg, Phys. Fluids B 5, 3969 (1993).

[8] J. Candy and M. N. Rosenbluth, Phys. Plasmas 1, 356 (1994).

[9] C. Z. Cheng, L. Chen, and M. S. Chance, Ann. Phys. 161, 21 (1985).

[10] L. Chen, Phys. Plasmas 1, 1519 (1994).

[11] C. Nührenberg, Phys. Plasmas 6, 137 (1999).

[12] A. Hasegawa and L. Chen, Phys. Fluids 19, 1924 (1976).

[13] C. Z. Cheng and M. S. Chance, Phys. Fluids 29, 3695 (1986).

[14] G. Y. Fu and J. W. V. Dam, Phys. Fluids B 1, 1949 (1989).

[15] A. Fasoli, A. Jaun, and D. Testa, Phys. Lett. A 265, 288 (2000).

[16] D. Testa, T. Panis, P. Blanchard, A. Fasoli, and JET-EFDA contributors, Nucl. Fusion 52, $094006(2012)$.

[17] H. Qin, W. M. Tang, and G. Rewoldt, Phys. Plasmas 6, 2544 (1999).

[18] P. Lauber, S. Günter, and S. D. Pinches, Phys. Plasmas 12, 122501 (2005).

[19] P. Lauber, S. Günter, A. Könies, and S. D. Pinches, J. Comp. Phys. 226, 447 (2007).

[20] A. Mishchenko, R. Hatzky, and A. Könies, Phys. Plasmas 15, 112106 (2008).

[21] A. Mishchenko, A. Könies, and R. Hatzky, Phys. Plasmas 16, 082105 (2009).

[22] A. Mishchenko, A. Könies, and R. Hatzky, Phys. Plasmas 18, 012504 (2011).

[23] Y. Nishimura, Phys. Plasmas 16, 030702 (2009).

[24] I. Holod, W. L. Zhang, Y. Xiao, and Z. Lin, Phys. Plasmas 16, 122307 (2009).

[25] T. S. Hahm, W. W. Lee, and A. J. Brizard, Phys. Fluids 31, 1940 (1988). 
[26] C. de Boor, A Practical Guide to Splines (Springer-Verlag, New York, 1978).

[27] K. Höllig, Finite Element Methods with B-Splines (Society for Industrial and Applied Mathematics, Philadelphia, 2003).

[28] M. Fivaz, S. Brunner, G. de Ridder, O. Sauter, T. M. Tran, J. Vaclavik, L. Villard, and K. Appert, Comp. Phys. Commun. 111, 27 (1998).

[29] A. Mishchenko, R. Hatzky, and A. Könies, Phys. Plasmas 11, 5480 (2004).

[30] A. Mishchenko, A. Könies, and R. Hatzky, in Proc. of the Joint Varenna-Lausanne International Workshop (Società Italiana di Fisica, Bologna, 2004).

[31] A. Mishchenko, A. Könies, and R. Hatzky, Phys. Plasmas 12, 062305 (2005).

[32] R. Hatzky, A. Könies, and A. Mishchenko, J. Comp. Phys. 225, 568 (2007).

[33] W. W. Lee, J. Comp. Phys. 72, 243 (1987).

[34] R. Hatzky, T. M. Tran, A. Könies, R. Kleiber, and S. J. Allfrey, Phys. Plasmas 9, 898 (2002).

[35] Y. Chen and S. Parker, J. Comp. Phys 189, 463 (2003).

[36] A. Könies, S. Briguglio, N. Gorelenkov, T. Fehér, M. Isaev, P. Lauber, A. Mishchenko, D. A. Spong, Y. Todo, W. A. Cooper, R. Hatzky, R. Kleiber, M. Borchardt, G. Vlad, ITPA EP TG, in Proc. of 24th Fusion Energy Conf. (IAEA, Vienna, 2012).

[37] T. Fehér, Ph.D. thesis, Max-Planck-Institut für Plasmaphysik, Greifswald, 2013.

[38] Y. Todo, Phys. Plasmas 13, 082503 (2006).

[39] S. Briguglio, G. Fogaccia, G. Vlad, F. Zonca, K. Shinohara, M. Ishikawa, and M. Takechi, Phys. Plasmas 14, 055904 (2007).

[40] V. Kornilov, R. Kleiber, R. Hatzky, L. Villard, and G. Jost, Phys. Plasmas 11, 3196 (2004).

[41] M. S. Chu, J. M. Green, L. L. Lao, A. D. Turnbull, and M. S. Chance, Phys. Fluids B 4, 3713 (1992).

[42] A. Könies and R. Kleiber, Phys. Plasmas 19, 122111 (2012). 


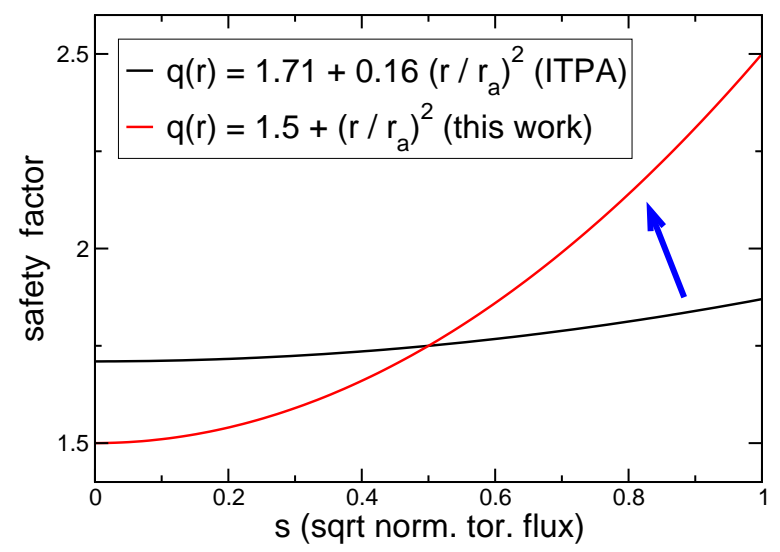

FIG. 1: (Color online) Safety factor profile used in this paper vs. the low-shear profile implemented in Refs. [21, 36] ("ITPA benchmark" case). 


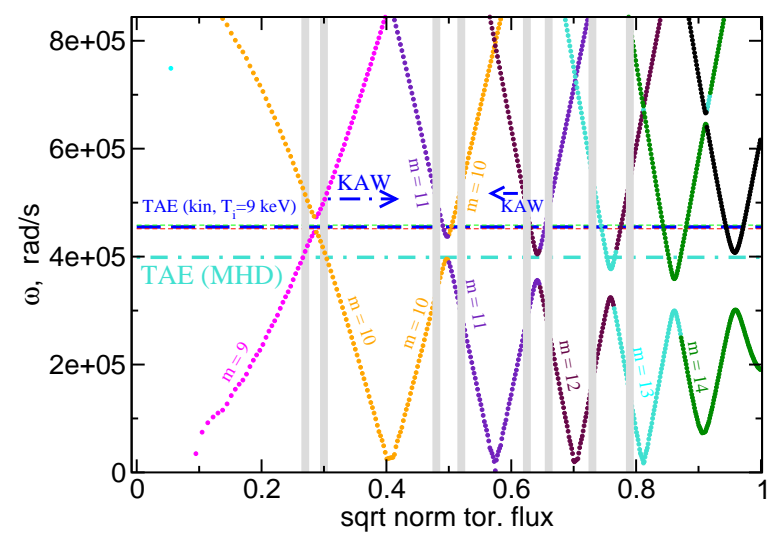

FIG. 2: (Color online) Reduced ideal MHD shear Alfvén continuum (includes the frequency upshift corresponding to $T_{i}=T_{e}=9 \mathrm{keV}$ ). One can see the toroidal gap corresponding to the coupled poloidal mode numbers $m_{1}=10$ and $m_{2}=11$. The frequencies of the MHD TAE mode and the gyrokinetic TAE mode (at $T_{i}=T_{e}=9 \mathrm{keV}$ ) are also shown. The gyrokinetic TAE mode can interact with the continuum at a number of locations (marked with grey vertical lines). Here, the Kinetic Alfvén Waves can be excited. 


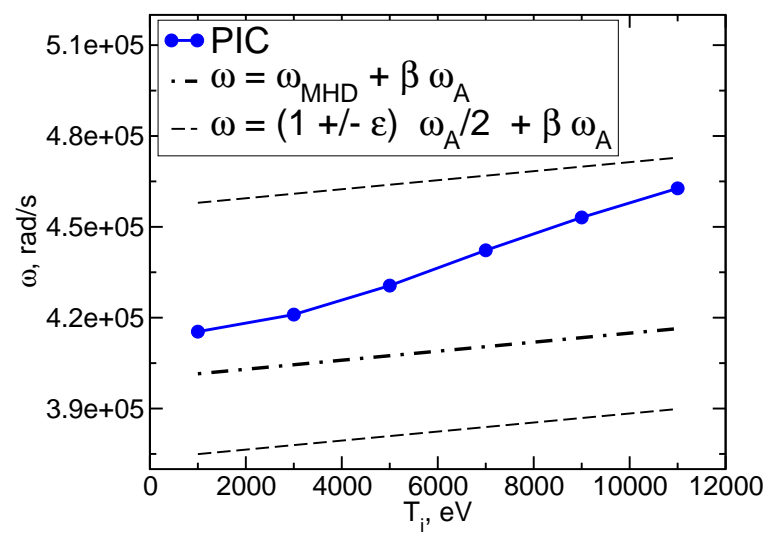

FIG. 3: (Color online) bulk plasma temperature effect on the TAE mode frequency. Here, the bulk plasma temperature $T_{i}=T_{e}$, the fast-particle temperature $T_{f}=0.4 \times 10^{6} \mathrm{eV}$ and the fast ion density is given by $n_{f 0}=1.5 \times 10^{17} \mathrm{~m}^{-3}$ [see Eq. (8)]. The acoustic modification of the SAW spectrum is indicated with the black lines (corresponding to the gap boundaries and the ideal eigenfrequency upshift). 


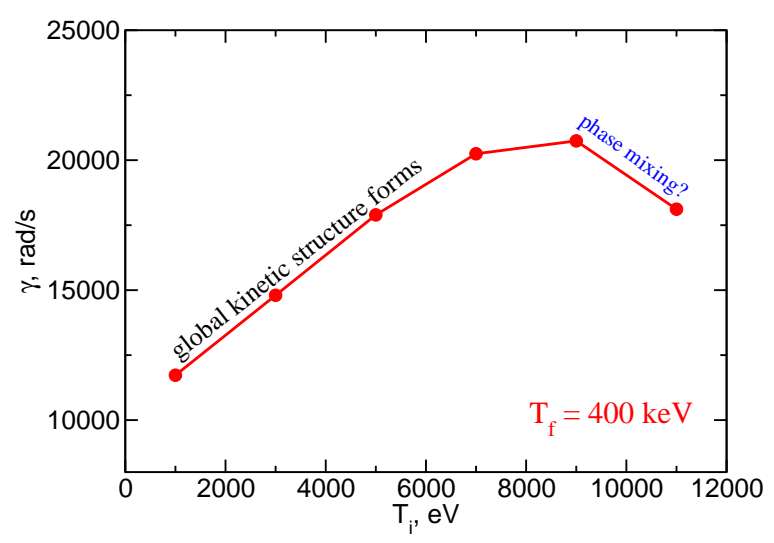

FIG. 4: (Color online) bulk plasma temperature effect on the TAE mode growth rate. The mode is more unstable at higher $T_{i}$ since a global kinetic (K)TAE-KAW structure builds up which is more efficient in tapping the fast ion free energy than the mere TAE mode. Here, the fast ion temperature $T_{f}=0.4 \times 10^{6} \mathrm{eV}$ and the fast ion density computed at $s=0.5$ is $n_{f 0}=1.5 \times 10^{17} \mathrm{~m}^{-3}$ [see Eq. (8)]. There is a competition between the stabilising phase-mixing effect and destabilising fast particles. Such a trade-off may be responsible for the relative stabilisation at $T_{i}>9 \mathrm{keV}$ although a detailed understanding of the associated physics is beyond the scope of the numerical study here. 

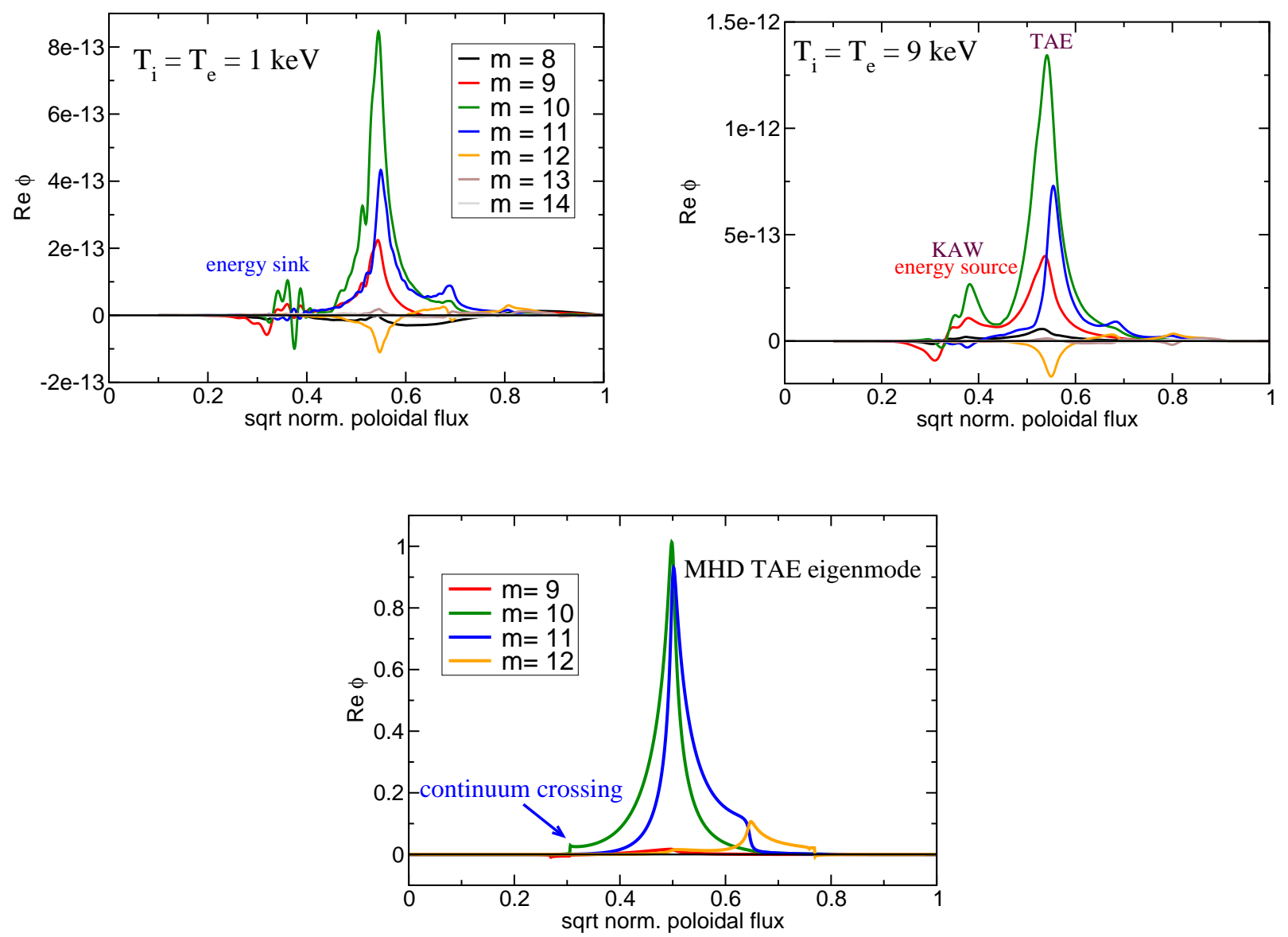

FIG. 5: (Color online) The gyrokinetic mode structure at two different bulk plasma temperatures. The global unstable (K)TAE-KAW structure forms at $T_{i}=T_{e}=9 \mathrm{keV}$ (on the right). In this case, the KAW part of the structure is destabilised by the fast ions which leads to larger growth rates of the global "compound" mode (compared to a single TAE with an energy sink at the continuum-resonance position). The same fast ion parameters were used for both simulations. For comparison, the ideal reduced-MHD TAE eigenmode is also shown. 


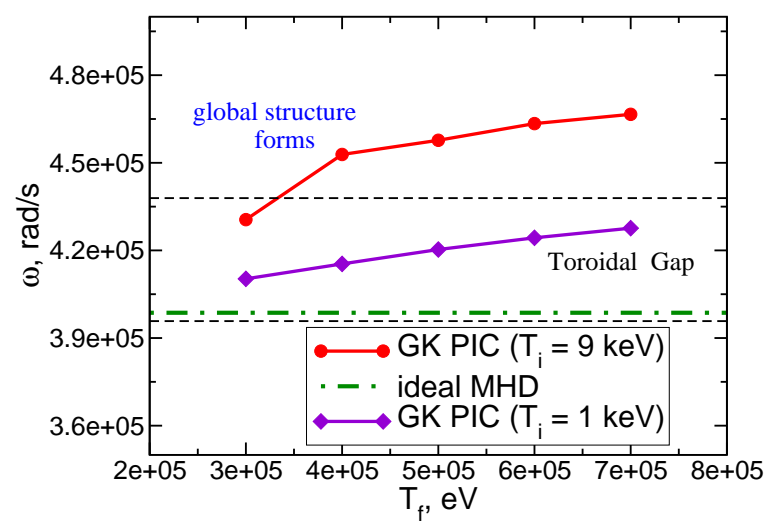

FIG. 6: (Color online) fast ion temperature scan at fixed fast ion density $n_{f 0}=1.5 \times 10^{17} \mathrm{~m}^{-3}$ and the bulk plasma temperature (the cases with $T_{i}=T_{e}=9 \mathrm{keV}$ and $T_{i}=T_{e}=1 \mathrm{keV}$ have been considered). The ideal-MHD frequency computed neglecting the acoustic effect is shown for comparison (this frequency has been used in the hybrid-gyrokinetic simulations, see Fig. 7). The toroidal gap shown between the dashed lines is measured at $s=0.5$ and corresponds to $T_{i}=T_{e}=9 \mathrm{keV}$. 


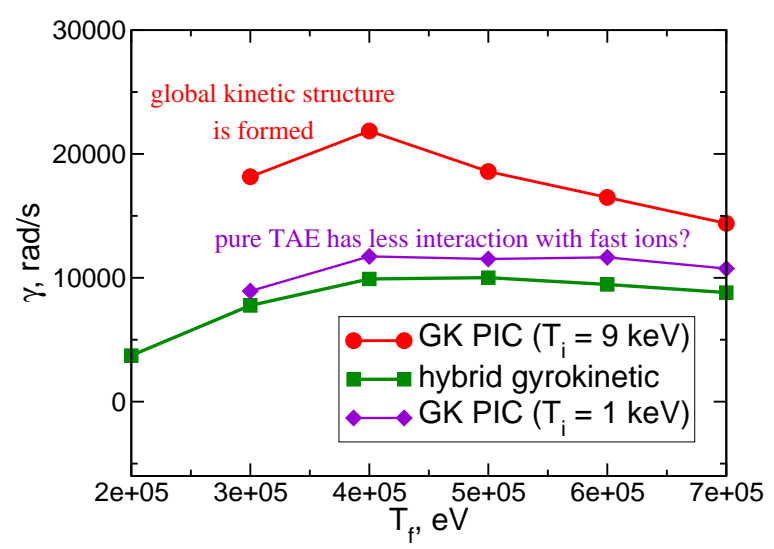

FIG. 7: (Color online) fast ion temperature scan at fixed fast ion density $n_{f 0}=1.5 \times 10^{17} \mathrm{~m}^{-3}$ and the bulk plasma temperature (corresponding to the cases with $T_{i}=T_{e}=9 \mathrm{keV}$ and $T_{i}=T_{e}=$ $1 \mathrm{keV})$. The dependencies of the gyrokinetic growth rates are compared with the hybrid-gyrokinetic computation $[37,42]$. The dependence at the low bulk plasma temperature agrees well with the gyrokinetic result, in contrast to the high bulk plasma temperature case. 


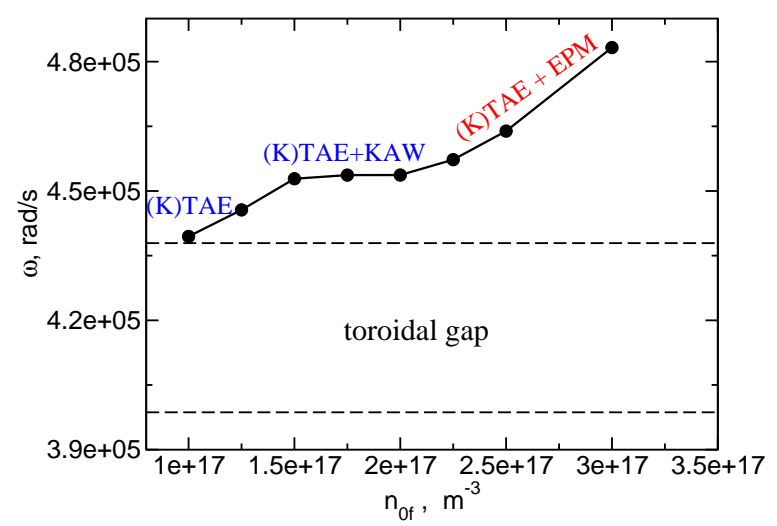

FIG. 8: (Color online) The frequency of the global mode is shown as a function of the fast ion density. Here, $T_{i}=T_{e}=9 \mathrm{keV}$ and $T_{f}=400 \mathrm{keV}$. The ideal-MHD gap boundaries are computed at $s=0.5$ for the consistent bulk plasma pressure but neglecting the pressure of the fast ions. 


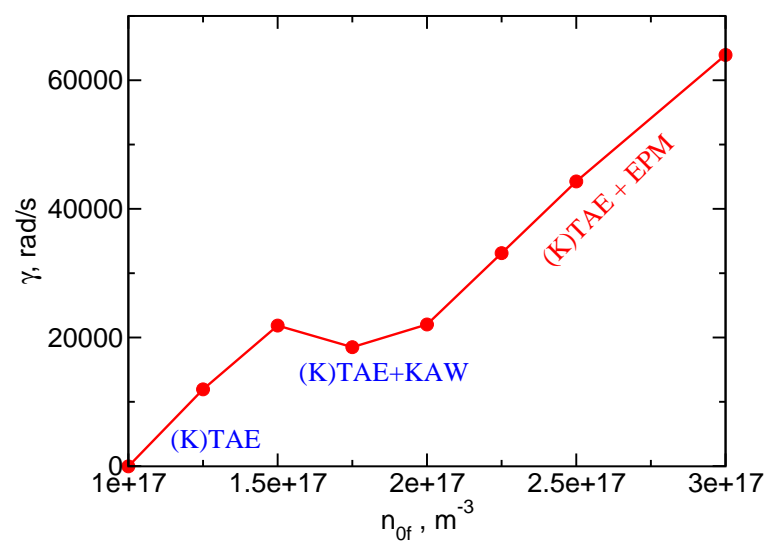

FIG. 9: (Color online) The growth rate of the global mode is shown as a function of the fast ion density. Here, $T_{i}=T_{e}=9 \mathrm{keV}$ and $T_{f}=400 \mathrm{keV}$. The (K)TAE mode coupled to the KAW transforms into the strongly unstable global (K)TAE-EPM structure when the fast ion density increases. This process is illustrated with the corresponding eigenmode structures shown in Fig. 10. 

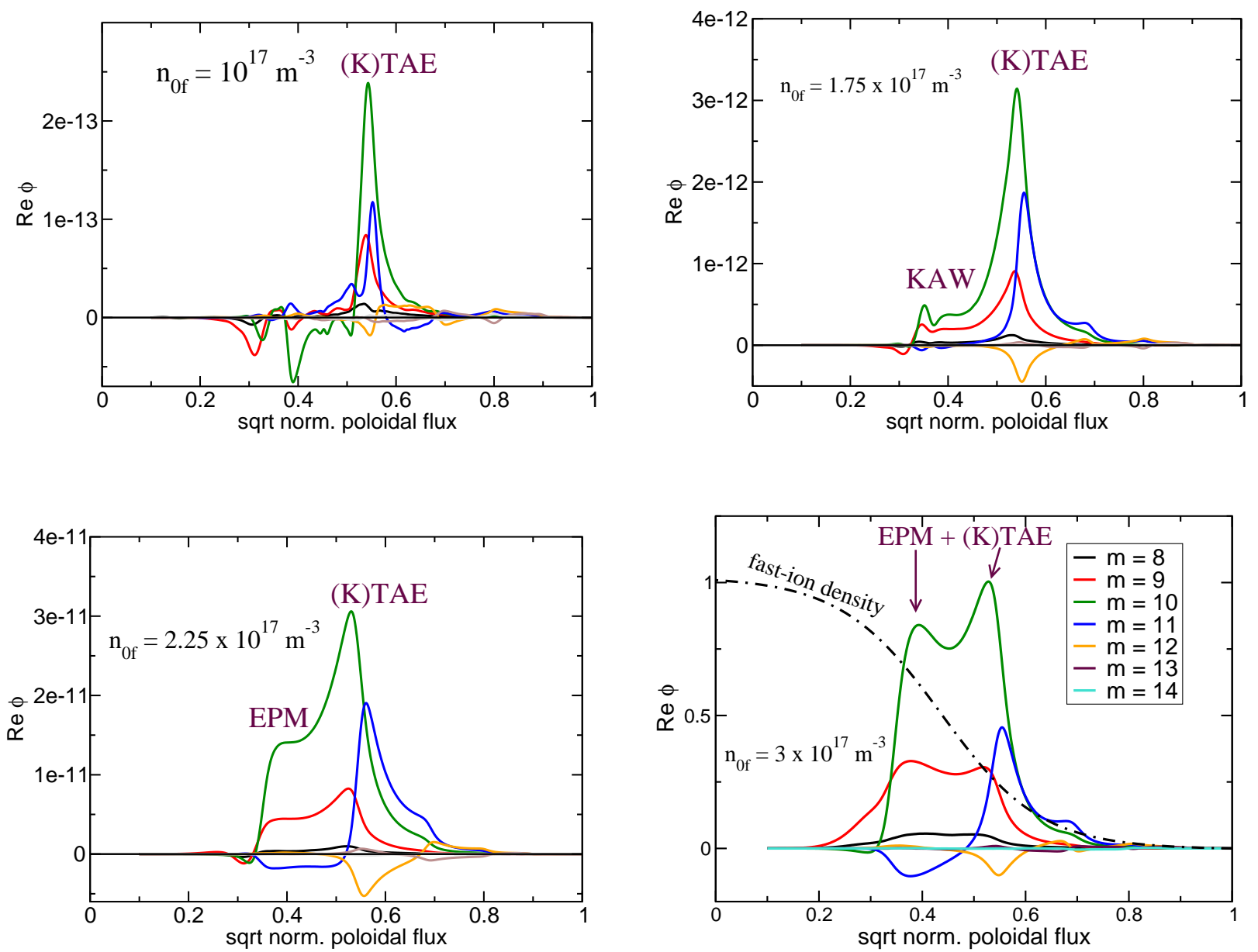

FIG. 10: (Color online) (K)TAE + KAW/EPM global mode structure appearing at different fast ion densities [see Eq. (8)]. The fast ion temperature $T_{f}=400 \mathrm{keV}$, the bulk plasma temperature $T_{i}=T_{e}=9 \mathrm{keV}$. A sketch of a fast ion density profile is shown, too. 


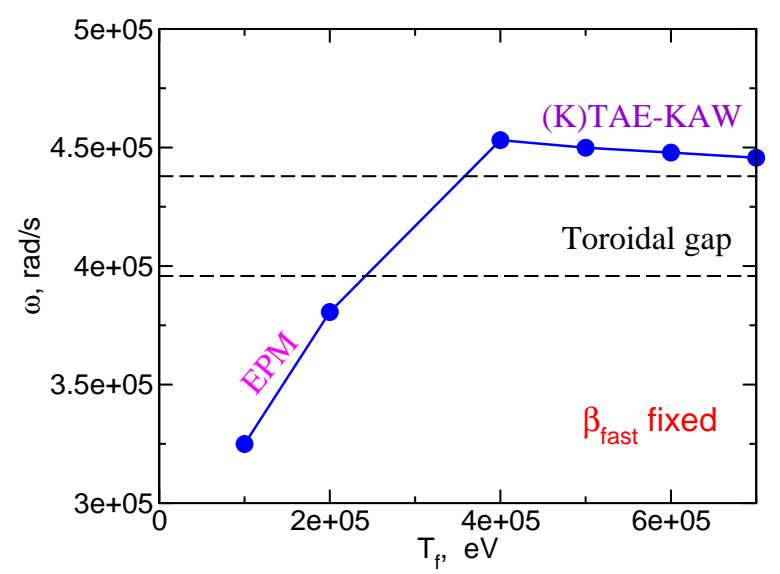

FIG. 11: (Color online) The frequency of the global mode as a function of the fast ion temperature at fixed $\beta_{\text {fast }}=0.0027$ and $T_{i}=T_{e}=9 \mathrm{keV}$. There are two distinct branches: the coupled (K)TAEKAW branch at higher fast ion temperatures (i.e. lower fast ion densities) and the "pure" EPM mode at the lower temperatures (which correspond to higher fast ion densities). 


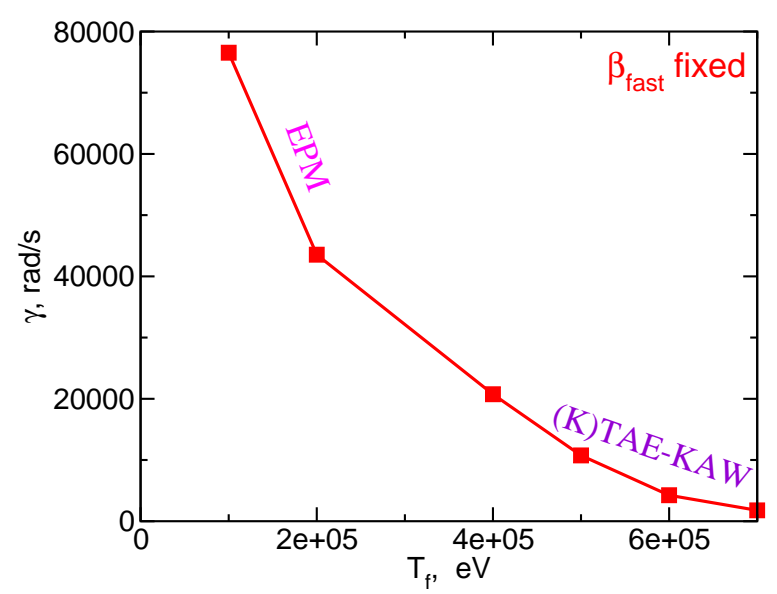

FIG. 12: (Color online) The growth rate of the global mode as a function of the fast ion temperature at fixed $\beta_{\text {fast }}=0.0027$ and $T_{i}=T_{e}=9 \mathrm{keV}$. The EPM mode is strongly driven since the radial location of the corresponding fast ion resonance is in the region with a large fast ion density gradient. 


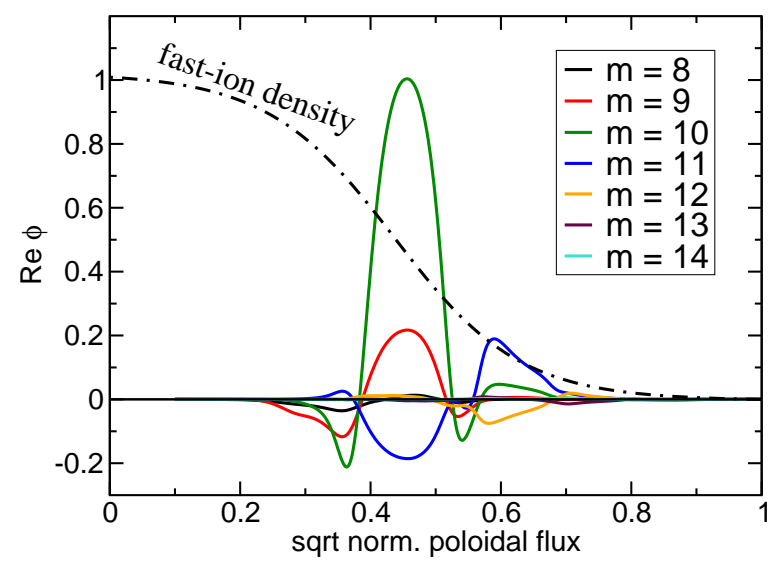

FIG. 13: (Color online) A "pure" EPM eigenmode structure (single-peak, cf. Fig. 10) corresponding to $T_{f}=200 \mathrm{keV}$ and $n_{0 f}=3 \times 10^{17} \mathrm{~m}^{-3}$. The bulk plasma temperature $T_{i}=T_{e}=9 \mathrm{keV}$. A sketch of a fast ion density profile is also shown [see Eq. (8)]. The "pure" EPM mode dominates since the drive of the $(\mathrm{K})$ TAE mode is too week for the parameters considered (low $T_{f}$ ). 